\title{
CFD Analysis of SVOC Mass transfer in Different Chambers
}

\author{
Yun-Feng MAO ${ }^{1}$, Zhuo $\mathrm{LI}^{1}$, Ya-Ling $\mathrm{HE}^{2}$ and Wen-Quan $\mathrm{TAO}^{1,2,{ }^{*}}$; \\ ${ }^{1}$ State Key Laboratory of Pollution Control and Resources Reuse, College of \\ Environmental Science \& Engineering, Tongji University, Shanghai 200092, China \\ ${ }^{2}$ Key Laboratory of Thermo-Fluid Science and Engineering of MOE, School of Energy \\ and Power Engineering, Xi'an Jiaotong University, Xi'an 710049, China \\ *Corresponding email: wqtao@mail.xjtu.edu.cn
}

\begin{abstract}
Semi-volatile organic compound (SVOC) in indoor environment is an important research topic because of their wide use and persistent effect on human health. SVOC chambers have been continually improved to study the mass transfer characteristics in indoor environment. To avoid experimental disadvantages, CFD method is used in the present paper to study the effect on mass transfer, especially on steady time by velocity field from the comparison of SVOC mass transfer in two different SVOC chambers. The results indicate that the variance of air flow in small range strongly affects the steady concentration and has no obvious effect on steady time. Sorption ability itself has great impact on steady time. The great reduction of steady time in Chamber B is also the combined effect of sorption and velocity field. The velocity field resulted from the special structure of Chamber B leads to a stronger convective mas transfer resistance, and hence causes a weaker effective sorption. Therefore, the less steady time in Chamber B is the result of weaker effective sorption besides a less sorption area of Chamber B than Chamber A.
\end{abstract}

KEYWORDS: SVOC; CFD; velocity field; sorption; Chambers 


\section{INTRODUCTION}

Semi-volatile organic compounds (SVOCs) are ubiquitous in the indoor environment, and can be found from things such as vinyl flooring, wall covering, floor tiles, furniture, and electronics [1]. A man keeps exposure to indoor air for about $90 \%$ time per day [2], and exposures to SVOCs can occur via inhalation, ingestion and dermal pathways, which straightly impact on human health. Studies $[2,3,4,5]$ suggest that exposure to semi-volatile organic compounds (SVOC) has been associated with adverse health effects such as asthma, rhinitis, allergy and reproductive toxicity. Especially for children, SVOCs even could relate to respiratory disease and growth in developing tissue, effect on the development of male reproductive tract, prenatal mortality, and reduce growth and birth weight [6]. Phthalate, as a main kind of SVOCs, has been widely used as plasticizers to enhance the flexibility of polyvinylchloride (PVC) products [7]. These phthalates include butyl decyl phthalate (BDP), $\operatorname{di}(2-$ ethylhexyl) phthalate(DEHP), di(n-octyl) phthalate(DNOP), diisooctyl phthalate(DIOP) and n-Octyl n-decyl phthalate(ODP), etc. Despite of the tremendous threat to human health, phthalates are produced at a large rate of several billion tons/yr. within a decade [8]. Therefore, understanding the transport and environmental fate of phthalates in the indoor environment is significant to human health.

Experiments in chambers are the main method to characterize the fate and transport of phthalates. Destaillats et al. (2008) [9] summarized some standard chambers designed for air pollutants, such as ozone, carbonyls, volatile organic compounds (VOCs) and particles. Among these chambers, the FLEC (field and laboratory 
emission cell) $[10,11,12]$ and CLIMPAQ (chamber for laboratory investigations of materials, pollution, and air quality) $[13,14,15]$ have been widely used to study the emission of VOCs. The research methods and experimental chambers on transport characteristics for SVOCs are inherited from those for VOCs. Clausen et al. [16] studied the characteristics of DEHP emission from PVC flooring and sorption on dusts in CLIMPAQ and FLEC. Xu and Little (2006) [17] developed a comprehensive mass transfer model of SVOCs in the indoor environment based on the experiments in the same two chambers. According to the SVOC model, Clausen et al. furtherly studied the influences of humidity [18] and temperature [19] on the emission and gaseous concentration. With increasing in-depth studies of SVOCs transport, the disadvantages of the experiments in these chambers become more obvious. For example, the time to steady state for VOCs is only several hours [20], but it takes more than a hundred of hours for SVOCs [19]. SVOCs have high boiling points and low saturated vapor pressures (between $10^{-14}$ and $10^{-4}$ atm) [21]. They hence release from the source in an extremely low concentration, and tend to sorb on indoor surface and particles. Some researchers indicated that surface sorption could influence the time to reach steady state [22, 23]. Xu et al. (2012) [23] thus developed a specially-designed chamber by maximizing the ratio of emission surface area to sorption surface area. The specially-designed chamber (Chamber A, Fig. 1(a)) effectively reduced the time to steady state for phthalates from 150 days in FLEC to less than 30 days. Liang and $\mathrm{Xu}$ (2014) [7] further improved the chamber (Chamber B, Fig. 1(b)) based on the same 
theory. The time to steady state for phthalates was sharply reduced to 5 days, which greatly saved the experimental time.

Although some achievements on research in chambers have been obtained, the properties of SVOCs strongly impede the further research. The low gaseous concentration in the chamber strongly affects the experimental accuracy. For example, the DEHP gaseous concentration in FLEC at room temperature is below $1 \mu \mathrm{g} / \mathrm{m}^{3}[18]$, and the concentration at a temperature of $55{ }^{\circ} \mathrm{C}$ is still only $91 \mu \mathrm{g} / \mathrm{m}^{3}$ [19]. The DEHP gaseous concentration emitted from the PVC flooring with a large content of more than $20 \%$ at room temperature is only $2.37 \mu \mathrm{g} / \mathrm{m}^{3}$ [7]. The long sampling time due to low gaseous concentration also has an impact on the experimental sensitivity and thus further in-depth experiments. Even in the specially-designed chamber (Chamber A in Figure 1), a sampling duration of $24 \mathrm{~h}$ is still needed by a sampling pump [7]. Despite those difficulties, some problems about SVOC transport are urgently needed to study. Additionally, the further specially-designed chamber (Chamber B in Figure 1) couldn't obtain such a tremendous reduction of time for gaseous SVOCs to reach steady state only because of its less sorption surface. The velocity field in the chamber could also play a significant role in it. Moreover, although sorption by the chamber surface is considered to have strong effect on the time to reach steady state, there is actually no detailed study ever focusing on it. At this point of time no efficient and effective experimental method exists to study the effect on the time to reach steady state by velocity field. Furthermore, the low experimental sensitivity resulted from low gaseous concentration and long sampling time couldn't allow comprehensive experiments upon 
the effects by surface sorption. Therefore, research conclusions by experiments are very limited. Thus CFD methods including FVM and lattice Boltzmann method (LBM) can be employed to study the mass transfer characteristics of SVOCs. Li et al. (2015) [24], Mu et al. (2015) [25] and Hussain et al. (2015) [26] adopt the technique of LBM to obtain some significant transport information, which greatly overcome the measurement difficulties in experiments. But for some macro transport problems and large-scale irregular calculation domain, FVM may still be the best approach.

Due to experimental difficulty, there is no study to research SVOC mass transfer characteristics from the perspective of velocity field. This paper used the tool of CFD to compare the velocity field in Chamber $\mathrm{A}$ and Chamber $\mathrm{B}$, and tried to find the intrinsic reason for sharp reduction of time to steady state from the perspective of velocity field. The simulated results are compared with reported experimental data. The effects by air flow and surface sorption were studied. Meanwhile, we also intended to reveal more mass transfer mechanism of SVOCs by comparing different transport characteristics in the two chambers.

\section{PHYSICAL/MATHEMATICAL MODELS AND NUMERICAL METHOD}

\subsection{Physical model}

The top views of Chamber A and Chamber B are shown in Figure 1 (a) and (b), respectively. The two chambers from the side view are the same (Figure 1 (c)). The chamber is made of stainless steel, and placed between vinyl flooring (VF) sheets. The 
two chambers have a height of nearly $2 \mathrm{~cm}$ and a diameter of $20 \mathrm{~cm}$ (Table 1). Some special measures are taken to ensure the chamber being completely sealed. The detailed measures and design can be seen in Wu et al. [27]. Fresh air comes in through the inlet and flows out through the outlet. The two VF sheets are the only source of SVOC. The SVOC emits from the VF sheets, and is dispersed in the air. Part of them is adsorbed onto the chamber wall. The other flows out with the air through the outlet. The air flow in the cavity is laminar. The specific parameters of flow and SVOC transport are shown in Table 1.

The whole mass transport process of SVOC is shown in Figure 2, which includes the process of emission from the floor, dispersion in the air and sorption on the wall. The diffusion within the source material could be ignored, because the emission of SVOCs from the floor is subject to "external" control [16]. Ekelund et al. [28, 29] indicated that a thin film existed on the emission surface, and the SVOCs concentration in the film was kept constant $[19,30]$. Therefore, in the CFD model the diffusion of SVOCs in the material is not considered, and the SVOCs is assumed to be emitted from a boundary with a constant concentration. Thus the emission surface is treated as a first kind boundary condition, and they enter into the simulation domain through the boundary.

\subsection{Mathematical model of air flow}

The air flow in the chamber can strongly impact the transport of SVOCs in the air.

For incompressible flow, the conservation equations for the continuity and momentum 
are as follows:

$\frac{\partial \rho}{\partial t}+\frac{\partial\left(\rho u_{j}\right)}{\partial x_{j}}=0$

$\frac{\partial\left(\rho u_{j}\right)}{\partial t}+\frac{\partial\left(\rho u_{i} u_{j}\right)}{\partial x_{j}}=-\frac{\partial p}{\partial x_{j}}+\frac{\partial}{\partial x_{j}}\left(\rho v \frac{\partial u_{j}}{\partial x_{j}}\right)$

where $\rho$ is the air density; $v$ is the air kinematic viscosity.

\subsection{Mathematical model of SVOC transport}

To reveal the emission characteristics of the entire process the transient convection-diffusion equation is used in the present study:

$\frac{\partial(\rho C)}{\partial t}+\frac{\partial\left(\rho u_{j} C\right)}{\partial x_{j}}=\frac{\partial}{\partial x_{j}}\left(\Gamma \frac{\partial C}{\partial x_{j}}\right)+S$

where $C$ is the mass concentration of SVOCs in the air and $\Gamma$ is the diffusion coefficient of SVOCs.

\subsection{Boundary/initial conditions and source term}

(a) The initial conditions: the initial velocity and the initial SVOC concentration in the chamber are given as follows

$v=0$

$C=0$

(b) Inlet: Air velocity is constant and the inflow air is clean:

$$
\begin{aligned}
& v=v_{\text {inlet }} \\
& C=C_{\text {inlet }}=0
\end{aligned}
$$

According to the air flow rate in Table 1 , the value of $v_{\text {inlet }}$ is $0.44 \mathrm{~m} / \mathrm{s}$ in Chamber 
A, and $0.26 \mathrm{~m} / \mathrm{s}$ in Chamber B.

(c) Outlet: zero gradients of air velocity and SVOC concentration in the direction normal to the outlet surface are given:

$$
\begin{aligned}
& \frac{\partial u_{j}}{\partial x_{j}}=0 \\
& \frac{\partial C}{\partial x_{j}}=0
\end{aligned}
$$

(d) Axis of symmetry: at the axis of symmetry, we have

$$
\begin{aligned}
& \frac{\partial u_{j}}{\partial x_{j}}=0 \\
& \frac{\partial C}{\partial x_{j}}=0
\end{aligned}
$$

(e) Wall: The emission boundary condition is

$$
\begin{aligned}
& u_{j}=0 \\
& C=C_{0}
\end{aligned}
$$

where $\mathrm{C}_{0}$ is the concentration in the source.

(f) Source term [30]

$$
S= \begin{cases}\frac{K_{s}\left(C_{M}^{t}-C_{M}^{t-1}\right)}{\Delta t} & \text { cells adjacent to the sorption wall } \\ 0 & \text { cells in the other domain }\end{cases}
$$

where $C_{M}^{t}$ is the concentration adjacent to the sorption wall at current flow time, $C_{M}^{t-1}$ is the concentration adjacent to the sorption wall at last flow time, $\Delta t$ is the time step.

\subsection{Numerical method}

The finite-volume method (FVM) based on commercial software Fluent 6.3 is employed to calculate the flow field and SVOC concentration. After the grid 
independency test is carried out, 1,410,308 grids in Chamber A and 630,519 grids in Chamber B are used, respectively. Because of the symmetry of the cavity, only half of the chambers are simulated. The SIMPLEC algorithm is adopted to deal with the pressure-velocity coupling. The second-order upwind scheme is used to discretize the convective terms and the central difference scheme for diffusion terms. For the transient DEHP transport equations, variable time step sizes are used to reduce the computation time with high accuracy. To calculate the SVOC concentration in the simulation domain, user-defined functions are used to define the process of sorption.

\section{RESULTS AND DISCUSSION}

\subsection{Validation of the present model}

The validity of the present model is conducted by comparing the simulation results with experimental data. The experimental data used to compare with the present model for Chamber A is from the experiment by Xu et al. (2012) [23], and the experimental data for the new SVOC chamber is from the experiment by Liang and Xu (2014) [7]. The validation results are shown in Fig. 3 (Chamber A in Fig. 3(a); Chamber B in Fig. 3(b)). In Fig. 3, the simulated results agree well with the experimental results, indicating the reliability of the present simulation model.

\subsection{Effect of air flow}

Liang and $\mathrm{Xu}$ (2015) [31] studied the effect of airflow on gaseous DEHP concentration by Chamber B. They found that the concentration at steady state 
decreased with rising air flow rate and the time to reach steady state varied with air flow. In fact, it is easy to understand that the concentration decreases when air flow increases, because the emission rate is quite low compared with airflow rate. A larger air flow could dilute the concentration in the room air. The effects of air flow rate on the time to reach a steady state are multi-fold. A large airflow tends to cause a low concentration, and thus it could be imagined that the time to reach steady state could decrease with increasing air flow rate. However, when considering wall sorption, a large airflow could lead to a large emission rate. A quick change of gaseous concentration further results in a relatively stronger sorption, which increases the time to reach steady state. The conclusion is not available in the study by Liang and $\mathrm{Xu}$ (2015) [31] because of the long sampling duration in the experiments. Therefore, by the present CFD model, we compared the cases with different airflows. Three cases were numerically tested: the case included in Table 1, the double airflow and the half airflow cases. The results are shown in Figure 4.

As we can see in Figure 4, when the airflow increases, the concentration at steady state greatly decreases. In Chamber B, the steady concentration at airflow of $Q / 2$ even reaches twice times than it at the airflow of $2 Q$. This result is in good agreement with Liang and $\mathrm{Xu}$ (2015) [31] and $\mathrm{Xu}$ and Little (2006) [17]. The steady concentration change in Chamber B is a little less than that in Chamber A, it's because Chamber B has a less chamber volume when they have the same inflow. As far as the time to reach the steady state (the steady time thereafter) is concerned, it also varies with the airflow. The larger the airflow rate the shorter the time. And this is more obvious in Chamber B. 
As indicated above, the steady time is the combined effects of airflow rate and wall sorption, larger airflow tends to reduce the steady time, but a stronger sorption rate tends to increase the steady time. Therefore, the opposite two effects lead to a less significant change of steady time with airflow rate than the change of steady concentration.

\subsection{Comparison of SVOC mass transfer in two different chambers}

It is widely agreed that surface sorption could affect the time to reach steady state $[22,23]$. For the two different chambers the concentration at different time points with sorption and without sorption are compared to investigate the effect of sorption. Additionally, we can speculate that the velocity field plays an important role in it. Thus both the effects of sorption and the velocity fields in the two chambers are compared.

\subsubsection{Comparison of sorption effect}

As we can see in Figure 4, the SVOC concentration increases quickly during the initial 20 days in Chamber A (a) and the initial 5 days in Chamber B (b), respectively. We further compare the concentration in different time range. Figure 5(a), Figure 5(b) and Figure 5(c) are the concentration changes with time in the first minute, the first hour and the first day, respectively. Figure 6 is the contour of the volume-average concentration in the two chambers at different time.

In Figure 5(a), the concentration grows quickly during the initial 20 seconds in

Chamber A and during the initial 30 seconds in Chamber B. After it, the concentration 
in Chamber B is slightly lower than it in Chamber A. Both of them have the same rising trend. After 30 minutes (Figure 5(b)), the concentration in Chamber B is higher than it in Chamber A. In the initial hour, the concentration accumulation mainly occurs in the first minute. From Figure 5(c), we can easily find that the concentration in Chamber $\mathrm{B}$ has a higher rising rate. The three figures generally indicate that the difference of the increasing rate is apparent in long period. From the concentration contour (Figure 6), we can find the obvious sorption effect on the sorption wall at different time. At 1 minute and 1 hour, there is an obvious low concentration on the sorption surface and a large concentration gradient normal to the sorption wall, which indicates that sorption process is relatively stronger at the time. But at 1 day (Figure $6 c)$, the sorption is very weak due to very weak concentration gradient normal the sorption wall. To exam the effect by sorption, we further compare the concentration change without sorption (Figure 7) and with different values of sorption surface/air partition coefficient (Figure 8).

From Figure 7, we can find that surface sorption has tremendous effect on the time to reach steady state. Without sorption, the concentration in both chambers can reach steady state in several minutes. It gets steady state at 150 seconds in Chamber A and 100 seconds in Chamber B, respectively. The difference is quite small contrast with that including sorption, which are 5 days and 20 days, respectively. Without sorption, SVOCs actually behaves like VOCs. Compared with SVOCs, VOCs have low boiling point and hence are difficult to adsorb on wall surface. As we have pointed out above that sorption has tremendous effect on the steady time, VOCs only need several hours 
to reach steady state [20], much less than several days for SVOCs. But differently the steady time for VOCs is still much longer than it for SVOCs (hundreds seconds) when not considering sorption. It is because the steady concentration of VOCs (nearly 5000 $\mu \mathrm{g} / \mathrm{m}^{3}$ ) is thousands times than the SVOC steady concentration (nearly $1 \mu \mathrm{g} / \mathrm{m}^{3}$ ). A larger steady concentration needs more time to reach it. Furthermore, VOCs emission is controlled by internal diffusion in the emission material [20. 32] while SVOCs emission is control by external convection in the boundary layer adjacent to the emission surface [16]. Thus the diffusion in the material strongly affects the time to get steady state in the air. Different from that, the diffusion in the material for SVOCs is neglected, and the emission of SVOC from a building material is considered to be emitted from a source with a constant concentration. Therefore, it is another main reason for SVOCs to get steady state much faster than VOCs when surface sorption is not considered.

Figure 8 shows the concentration with different sorption ability in the two chambers. No matter the sorption ability is weak or strong, it takes much longer time to reach steady state compared with mass transfer without sorption. The sorption capacity has little effect on the steady concentration. Because the two chambers have the same inflows, SVOC emission sources and surface/air sorption coefficients, the only difference is the velocity fields resulted from their different structures, hence we can infer that differences in concentration variation between Chamber A and B are resulted by different velocity fields. In the following sector, we study the effect by the velocity field. 


\subsubsection{Comparison of velocity field effect}

For the passive techniques of enhancing convective heat transfer, there are three types of enhanced methods [33]: i) increasing the interruption in the fluids; ii) decreasing the thermal boundary layer thickness; iii) increasing the velocity gradient near a heat transfer wall. Similar to heat transfer, mass transfer enhancement could also be explained by the three methods. The studied two chambers are on the same operation condition (inflow and temperature), and their walls have the same sorption strength $\left(K_{s}\right)$. Therefore, velocity field in the chamber is one of significant causes to effect the steady time, and velocity field may work along with wall sorption.

There are two methods of flow manipulation: alterations to the main flow and the introduction of secondary flows. The concentration distribution in the air is intimately coupled to the velocity field [34]. The velocity fields on the middle plane of the two chambers are shown in Figure 9. Chamber A and Chamber B have similar inlet velocity, but the geometries of the chambers, the number and location of inlets and outlets are totally different. All of those would lead to totally different velocity field, as shown in Figure 9. In chamber A, the inlet and the outlet are installed on the same line. But it is not the case with the Chamber B. In Chamber B, the flow gets disrupted by the wall near the outlet. In fact, there is no direct obstruction in Chamber B, but the different location of outlets makes the wall work as an obstruction. The constructed geometry causes swirl flows. Local mass-transfer enhancement could occur because of enhanced mass mixing induced by vortices, because the swirl flow could provide increased 
mixing to break up the boundary layer, increase the addition of another flow stream to the main flow stream and create a curved flow path. To further analyze the effect of velocity field, it is necessary to further compare the velocity gradient. The velocity gradient on the sorption wall is shown in Figure 10. In Figure 10, $L$ is the sorption surface length from the outlet at the location of $x=0$ to the other end, $\eta$ is the distance along the sorption surface from the outlet at the location of $x=0$.

In Figure 10, there is a relatively great velocity gradient near the outlet and the inlet in both chambers. On other sorption surface of Chamber A, the velocity gradient is very low. In Chamber B, there is a peak value near the outlet, because the inflow hits the opposite sorption wall. But from both Figure 9 and 10 there are many areas of low velocity gradient in Chamber B, they are at the corner of the chamber, between the inlets, and between the outlets. Therefore, swirl flows in Chamber B don't cause a substantial rise of velocity gradient. On the contrary, the special structure of Chamber B leads to a greatly lower velocity gradient on nearly $1 / 2$ of its sorption surface compared with Chamber A. Velocity gradient in other parts of sorption surface of Chamber B is similar with it in Chamber A, except the sorption surface hit by inflow. Lower velocity gradient on sorption surface of Chamber B could lead to a lower convective mass transfer coefficient. To analyze in detail the effects of velocity field on mass transfer, the local Sherwood number on the source surface at steady state is presented in Figure 11. The Sherwood number on the source surface is expressed in Equation (15) [35], $z$ is the normal direction to the source plane: 


$$
S h=\frac{\Delta z}{C-C_{b u l k}} \frac{\partial C}{\partial n_{1}}
$$

As we can see from Figure 11, the two chambers have similar range of local Sherwood number on the source surface (within 0-13). The local Sherwood number near their inlets, outlets and along the line from the inlet and outlet are relatively high. The Sherwood number distribution on the source surface is consistent with the velocity field (Figure 9). However, there are differences on the distribution of local Sh on their source surfaces. In Chamber A, nearly $2 / 3$ of its source surface is in a Sherwood number below 3. But in Chamber B, about $1 / 3-1 / 2$ of its surface area is in a Sherwood number below 3. Therefore, generally the emission on the source surface in Chamber B is subject to a slightly weaker mass transfer resistance than it in Chamber A, but their difference is not great.

Although secondary flow and vortex can help mass transfer, its effects are not obvious in the process of emission. The mass transfer on the sorption surface is further compared (Figure 12). Because at a minute as well as an hour strong sorption effect can be observed obviously from Figure 6, local Sherwood number on the sorption surface at a minute and an hour in the two chambers are compared, respectively (Figure 12). The local Sherwood number $S h$ on the sorption surface is defined:

$$
S h=\frac{\eta}{C_{0}-C} \frac{\partial C}{\partial n_{1}}
$$

In Figure 12 and Equation (16), $L$ is the sorption surface length from the outlet at the location of $x=0$ to the other end, $\eta$ is the distance along the sorption surface from the 
outlet at the location of $x=0$.

From the comparison of local Sherwood number on the sorption surface in the two chambers at 1 minute (Figure 12(a)), the local Sherwood numbers along the sorption surface in the two chambers are quite low and very similar, because the concentration at 1 minute is greatly low (compared with $C_{0}$ ) and could not lead to a large concentration gradient. The sorption mass transfer resistance in Chamber B is slightly lower than it in Chamber A, especially near the outlet and inlet. A lower mass transfer resistance indicates a more efficient sorption. That's why there is a slightly lower concentration in Chamber $\mathrm{B}$ than it in Chamber $\mathrm{A}$ at 1 minute. Additionally, a relatively larger Sherwood number is observed near the inlet and the outlet, because the air velocity is larger which can cause a thinner mass boundary thickness. In Figure 12(b), the local Sherwood number on the sorption surface in Chamber A is greatly larger than it in the Chamber B, and the difference could be as large as hundred times, which is consistent with the experimental results [7, 23]. Under the same operation condition in the two chambers, the convective mass transfer coefficient in Chamber A is $1.0 \times 10^{-2} \mathrm{~m} / \mathrm{s}\left(h_{s}\right)$ [23] while it in Chamber B is $2.1 \times 10^{-4} \mathrm{~m} / \mathrm{s}\left(h_{s}\right)$ [7]. The present authors further predicted the two convective mass transfer coefficients by the CFD simulations. The obtained values are $1.4 \times 10^{-2} \mathrm{~m} / \mathrm{s}$ in Chamber A, and $1.6 \times 10^{-4} \mathrm{~m} / \mathrm{s}$ in Chamber B, which are in the same order of magnitude with the experimental results shown above. The values of $h_{s}$ in Chamber B is as lower as two-hundred times than it in Chamber A, which is exactly consistent with the local Sherwood number in Figure 9 because the Sherwood number is proportional to the convective mass transfer 
coefficient. A lower convective mass transfer coefficient indicates weaker effective sorption ability. As we have discussed in Sector 3.3.1, lower sorption effect could greatly decrease the steady time. Therefore, surface sorption is a significant factor to affect the steady time, which is, in turn, affected by the velocity field in the chamber.

\section{CONCLUSION}

This research by CFD method deeply studied some SVOC transfer problems that traditional experiments were difficult to solve so far. The CFD results were compared with experimental data from existing papers. The focus was on the influence of airflow and sorption. The airflow has tremendous effect on steady concentration, but its effect on the steady time isn't obvious in both chambers. The different steady time in the two chambers is the result of combined effects by velocity field and sorption. Sorption ability has little effect on steady concentration, but its impact on steady time is obvious. Steady state even could be reached in minutes without sorption. The velocity field helps to raise the emission on the source in Chamber B, but its effect is not obvious. On the contrary, the velocity field has larger impact on sorption. The velocity field resulted from the special structure of Chamber B leads to a lower velocity gradient on large area of sorption surface in Chamber B. It also leads to a lower Sherwood number in Chamber B, which indicates a larger mass transfer resistance on sorption wall of Chamber B. All of them would cause a less effective sorption in Chamber. Because sorption strongly affects the steady time, the steady time in Chamber B is far less than it in Chamber A. 


\section{ACKNOWLEDGEMENT}

This work is supported by the $12^{\text {th }}$ Five-Year National Key Technology R \& D Program (2012BAJ02B03) and the National Science Foundation of China (Grant No. 51136004).

\section{REFERENCES}

[1] C. Bornehag, B. Lundgren, C. Weschler, T. Sigsgaard, L. Hagerhed-Engman, J. Sundell, Phthalates in indoor dust and their association with building characteristic, Environ. Health Perspect. 113 (2005) 1399-1404.

[2] J. Roberts, W. Nelson, National human activity pattern survey data base, United States Environmental Protection Agency (USEPA), Research Triangle Park, NC, 27-34, 1995.

[3] J. Jaakkola, H. Parise, V. Kislitsin, N. Lebedeva, J. Spengler, Asthma, wheezing, and allergies in russian schoolchildren in relation to new surface materials in the home, Am. J. Public Health, 94 (2004) 560-562.

[4] B. Kolarik, K. Naydenov, M. Larsson, C. Bornehag, J. Sundell, The association between phthalates in dust and allergic diseases among bulgarian children, Environ. Health Perspect. 116 (2008) 98-103.

[5] J. Lychea, A. Gutlebbd, A. Bergmanc, G. Eriksend, A. Murkef, E. Ropstada, M. Saundersg, J. Skaareda, Reproductive and developmental toxicity of phthalates, J. Toxicol., Env. Heal. B, 12 (2009) 225-249.

[6] C. Bornehag, E. Nanberg, Phthalate exposure and asthma in children, Int. J. Androl. 33 (2010) 333-345.

[7] Y. Liang, Y. Xu, Improved method for measuring and characterizing phthalate emissions from building materials and its application to exposure assessment, Environ. Sci. Technol. 48 (2014) 4475-4484.

[8] R. Rudel, L. Perovich, Endocrine disrupting chemicals in indoor and outdoor air, Atmos. Environ. 43 (2009) 170-181.

[9] H. Destaillats, R. Maddalena, B. Singer, A. Hodgson, T. Mckone, Indoor pollutants 
emitted by office equipment: a review of reported data and informatin needs, Atmos. Environ. 42 (2008) 1371-1388.

[10] E. Uhde, A. Borgschulte, T. Salthammer, Characterization of the field and laboratory emission cell - FLEC: Flow field and air velocities, Atmos. Environ. 32 (1998) 773-781.

[11] B. Jensen, P. Wolkoff, C. Wilkins, P. Clausen, Characterization of linoleum. Part 1: measurement of volatile organic compounds by use of the field and laboratory emission cell, "FLEC", Indoor Air 5 (1995) 38-43.

[12] R. Luo, J. Niu, Determining diffusion and partition coefficients of VOCs in cement using on FLEC, Build. Environ. 41 (2006) 1148-1160.

[13] L. Fang, G. Clausen, P. Fanger, Impact of temperature and humidity on chemical and sensory emissions from building materials, Indoor Air 9 (1999) 193-201.

[14] P. Blondeau, A. Tiffonnet, A. Damian, O. Amiri, J. Molina, Assessment of contaminant diffusivities in building materials from porosimetry tests, Indoor Air 13 (2003) 310-318.

[15] M. Zuraimi, C. Roulet, K. Tham, S. Sekhar, K. Cheong, N. Wong, K. Lee, A comparative study of VOCs in Singapore and European office buildings, Build. Environ. 41 (2006) 316-329.

[16] P. Clausen, V. Hansen, L. Gunnarsen, A. Afshari, P. Wolkoff, Emission of di-2-ethylhexyl phthalate from PVC flooring into air and uptake in dust: emission and sorption experiments in FLEC and CLIMPAQ, Environ. Sci. Technol. 38 (2004) 2531-2537.

[17] Y. Xu, J. Little, Predicting emissions of SVOCs from polymeric materials and their interaction with airborne particles, Environ. Sci. Technol., 40 (2006) $456-461$.

[18] P. Clausen, Y. Xu, V. Kofoed-Sørensen, J. Little, P. Wolkoff, The influence of humidity on the emission of di-(2-ethylhexyl) phthalate (DEHP) from vinyl flooring in the emission cell "FLEC", Atmos. Environ., 41 (2007) 3217-3224.

[19] P. Clausen, Z. Liu, V. Kofoed-Sørensen, J. Little, P. Wolkoff, Influence of temperature on the emission of di-(2-ethylhexyl) phthalate (DEHP) from PVC 
flooring in the emission cell FLEC, Environ. Sci. Technol. 46 (2012) 909-915.

[20] Y. Xu, Y. Zhang, An improved mass transfer based model for analyzing VOC emissions from building materials, Atmos. Environ. 37 (2003) 2497-2505.

[21] K. Hu, Q. Chen, J. Hao, Influence of suspended particles on indoor semi-volatile organic compounds emission, Atmos. Environ. 79 (2013) 695-704.

[22] P. Clausen, V. Hansen, A. Afshari, P. Wolkoff, Emission of phthalates from PVC flooring in two very different test chambers, Indoor Air 2 (2002) 932-937.

[23] Y. Xu, Z. Liu, J. Park, P. Clausen, J. Benning, J. Little, Measuring and predicting the emission rate of phthalate plasticizer from vinyl flooring in a specially-designed chamber, Environ. Sci. Technol. 46 (2012) 12534-12541.

[24] D. Li, Z. Tong, Y. He, W. Tao, Predicting the transport process of indoor semi-volatile organic compounds via lattice Boltzmann method, Build. Environ. 94 (2015) 82-96.

[25] Y. Mu, L. Chen, Y. He, W. Tao, Coupling finite volume and lattice boltzmann methods for pore scale investigation on volatile organic compounds emission process, Build. Environ. 92 (2015) 236-245.

[26] M. Hussain, Y. He, A. Mohamad, W. Tao, A new hybrid algorithm for numerical simulation of VOC emissions using single-layer and multilayer approaches, Numer. Heat T.R. B-Fund. 67 (2015) 211-230.

[27] Y. Wu, S. Cox, Y. Xu, Y. Liang, D. Won, X. Liu, P. Clausen, L. Rosell, J. Benning, Y. Zhang, J. Little, A reference method for measuring emissions of SVOCs in small chambers, Building and Environment 95 (2016) 126-132.

[28] M. Ekelund, B. Azhdar, M. Hedenqvist, S. Mikael, U. Gedde, Long-term performance of poly (vinyl chloride) cables, part 2: Migration of plasticizer, Polym. Degrad. Stab. 93 (2008) 1704-1710.

[29] M. Ekelund, B. Azhdar, U. Gedde, Evaporative loss kinetics of di (2-ethylhexyl) phthalate (DEHP) from pristine DEHP and plasticized PVC, Polym. Degrad. Stab. 95 (2010) 1789-1793.

[30] P. Clausen, Z. Liu, Y. Xu, V. Kofoed-Sørensen, J. Little, Influence of air flow rate on emission of DEHP from vinyl flooring in the emission cell FLEC: 
Measurements and CFD simulation, Atmos. Environ. 44 (2010) 2760-2766.

[31] Y. Liang, Y. Xu, The influence of surface sorption and air flow rate on phthalate emissions from vinyl flooring: Measurement and modeling, Atmos. Environ. 103 (2015) 147-155.

[32] Y. Xu, Y. Zhang, A general model for analyzing single surface VOC emission characteristics from building materials and its application, Atmos. Environ. 38 (2004) 113-119.

[33] W. Tao, Y. He, Q. Wang, Z. Qu, F. Song, A unified analysis on enhancing single phase convective heat transfer with field synergy principle, Int. J. Heat Mass Tran. 45 (2002) 4871-4879.

[34] A. Jacobi, R. Shah, Heat transfer surface enhancement through the use of longitudinal vortices: a review of recent progress, Exp. Therm. Fluid Sci. 11 (1995) 295-309.

[35] H. Eberl, C. Picioreanu, J. Heijnen, and M. Van Loosdrecht, A three-dimensional numerical study on the correlation of spatial structure, hydrodynamic conditions, and mass transfer and conversion in biofilms, Chem. Eng. Sci. 55 (2000) $6209-6222$. 
1. Table 1: Experimental conditions and model parameters 
Table 1: Experimental conditions and model parameters

\begin{tabular}{|c|c|c|}
\hline Parameter & $\begin{array}{c}\text { Chamber } \\
\text { A }\end{array}$ & $\begin{array}{l}\text { Chamber } \\
\text { B }\end{array}$ \\
\hline Temperature $\left({ }^{\circ} \mathrm{C}\right)$ & 22 & 25 \\
\hline Chamber volume (L), $V$ & 2 & 1 \\
\hline Air flow rate $(\mathrm{mL} / \mathrm{min}), Q$ & 850 & 1000 \\
\hline Air exchange rate $(1 / \mathrm{h})$ & 25 & 53 \\
\hline Area of test pieces $\left(\mathrm{m}^{2}\right), A$ & 0.252 & 0.13 \\
\hline Chamber height $(\mathrm{cm})$ & 2 & 1.8 \\
\hline Loading $\left(\mathrm{m}^{2} / \mathrm{m}^{3}\right)$ & 126 & 126 \\
\hline Concentration in equilibrium with vinyl flooring $\left(\mu \mathrm{g} / \mathrm{m}^{3}\right), C_{0}$ & 1.1 & 2.3 \\
\hline Sorption surface/air partition coefficient (m), $K_{s}$ & 1800 & 1500 \\
\hline
\end{tabular}


1. Figure 1. Configurations of the SVOC chambers; (a) old chamber; (b) new chamber; (c) side view of two chambers

2. Figure 2. Schematic representation of SVOC emissions in an experimental chamber

3. Figure 3. Comparison between simulation data and experimental data

4. Figure 4. Concentration variation affected by airflow

5. Figure 5. Non-dimensional concentration at different time

6. Figure 6. Non-dimensional concentration contour varying against time

7. Figure 7. SVOC concentration without sorption varying against time

8. Figure 8. The effect of Ks on non-dimensional concentration

9. Figure 9. Velocity field in SVOC chambers A and B, respectively

10. Figure 10. Velocity gradient on the sorption surface

11. Figure 11. Local Sherwood number on the source surface

12. Figure 12. Local Sherwood number on the sorption surface at (a) 1 minute and (b) 1 hour 


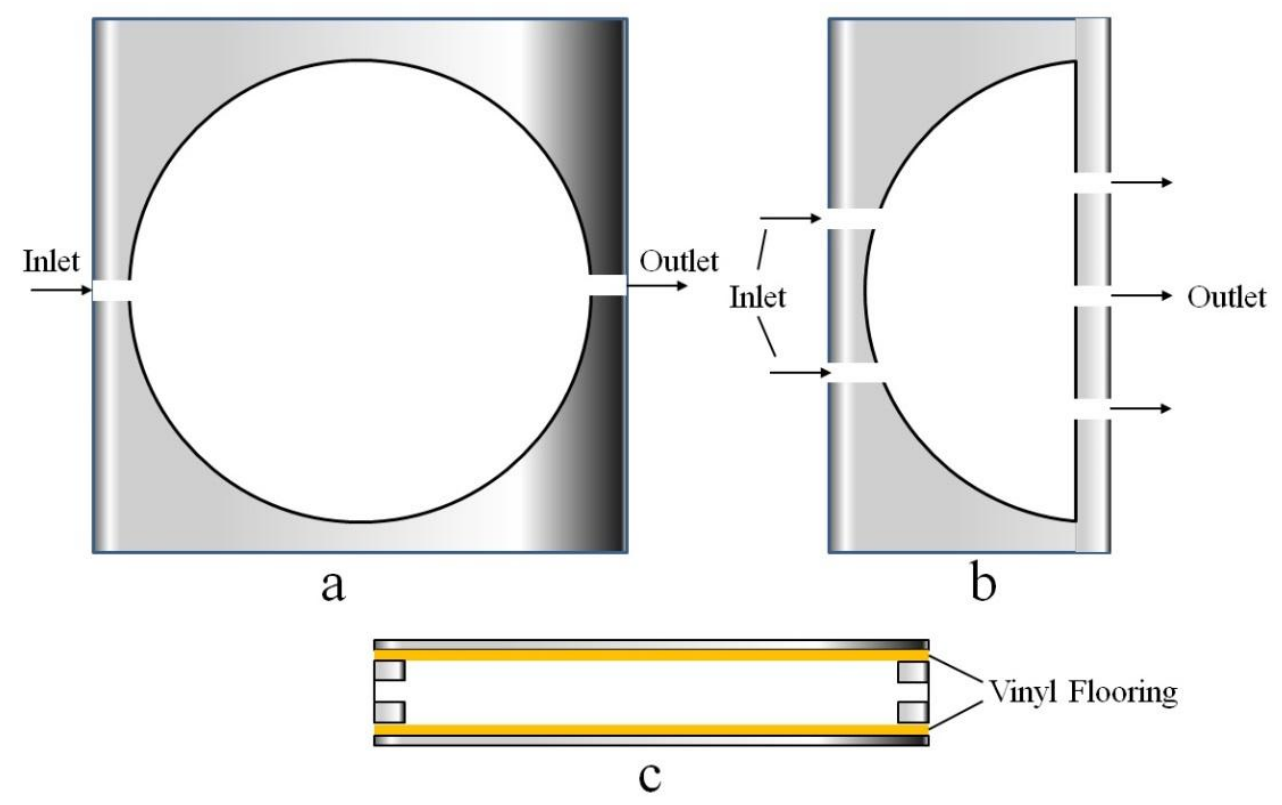

Figure 1. Configurations of the SVOC chambers; (a) Chamber A; (b) Chamber B; (c) side view of two chambers 


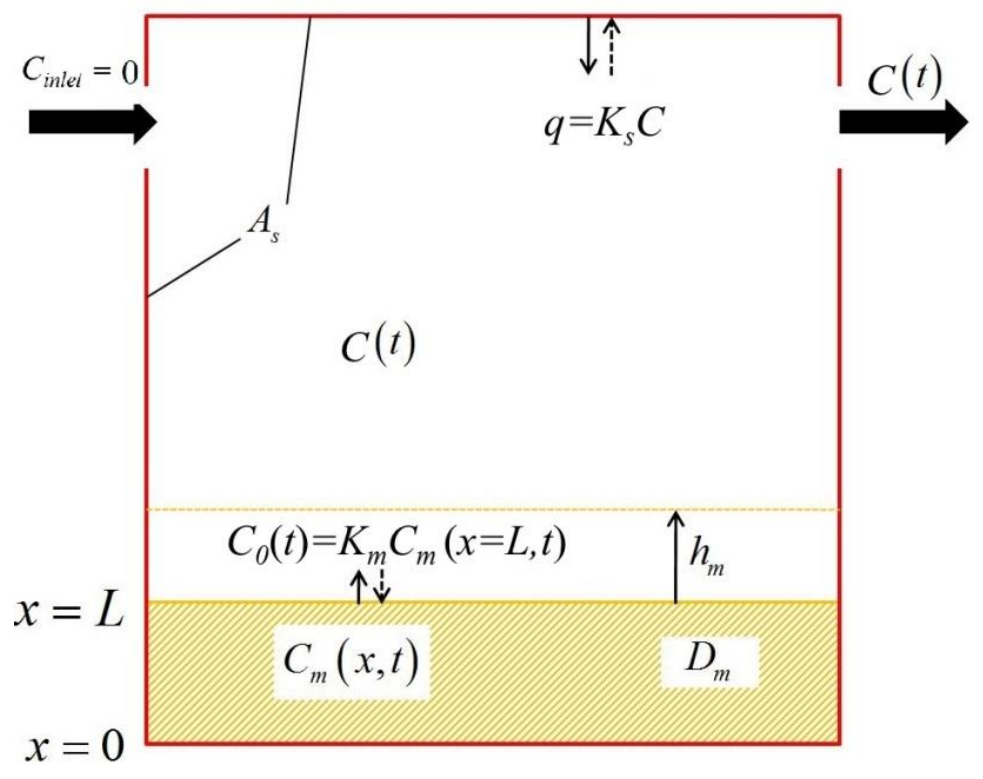

Figure 2. Schematic representation of SVOC emissions in an experimental chamber 


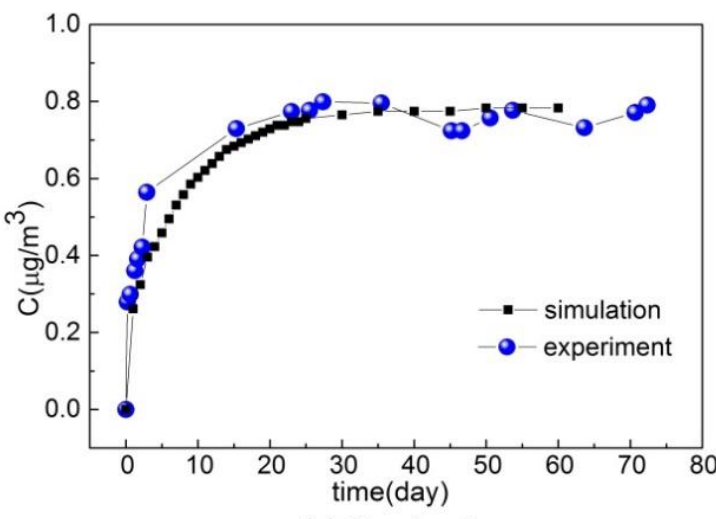

(a) Chamber A

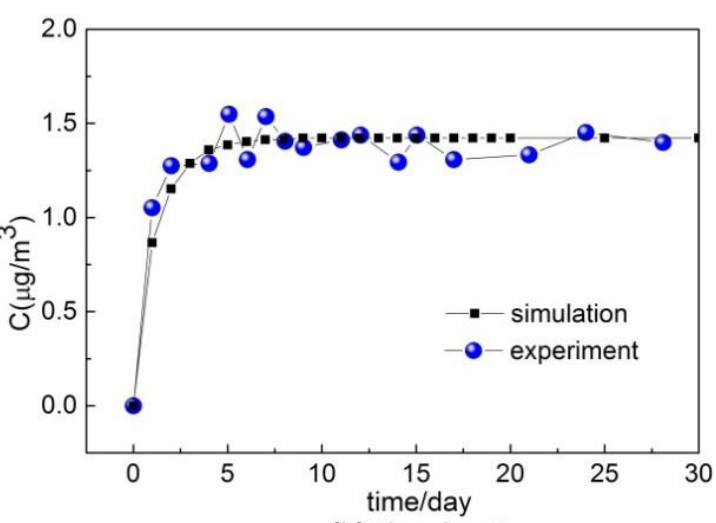

(b) Chamber B

Figure 3. Comparison between simulation data experimental data [7, 23] 




(a) the average $\mathrm{Cg}$ in Chamber $\mathrm{A}$

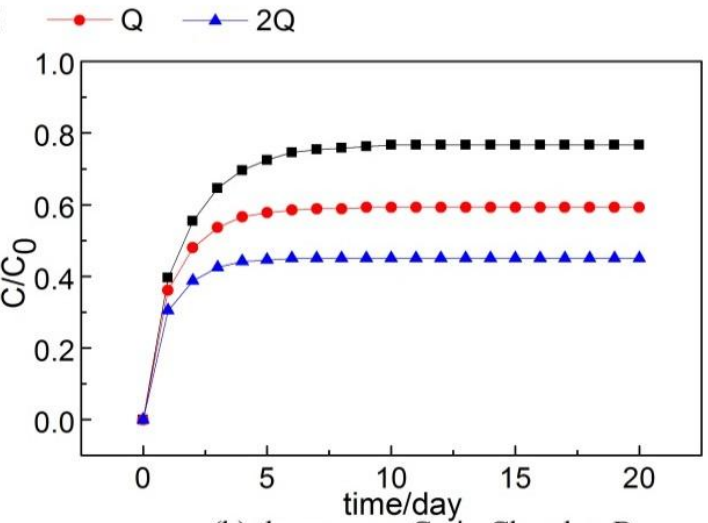

(b) the average $\mathrm{Cg}$ in Chamber $\mathrm{B}$

Figure 4. Concentration variation affected by airflow 


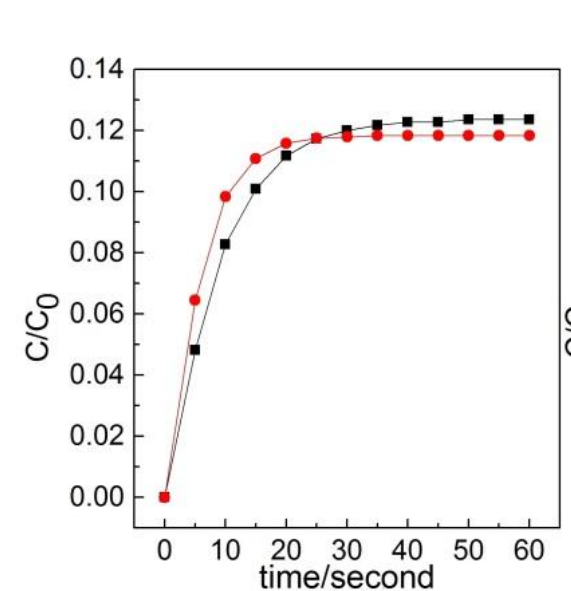

(a) the average $\mathrm{C}$ in first minute

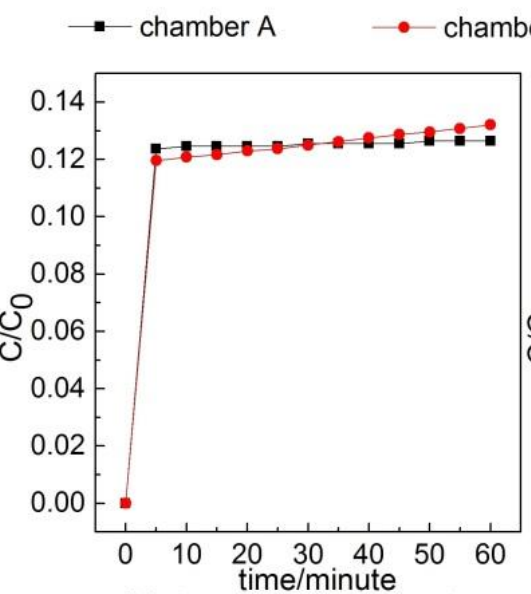

(b) the average $\mathrm{C}$ in first hour

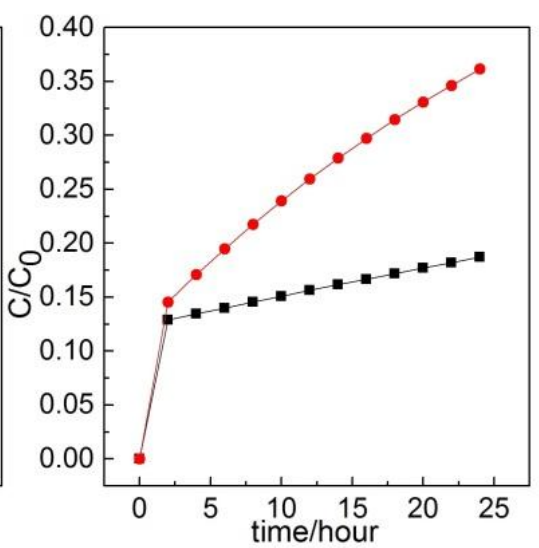

(c) the average $\mathrm{C}$ in first day

Figure 5. Non-dimensional concentration at different time 

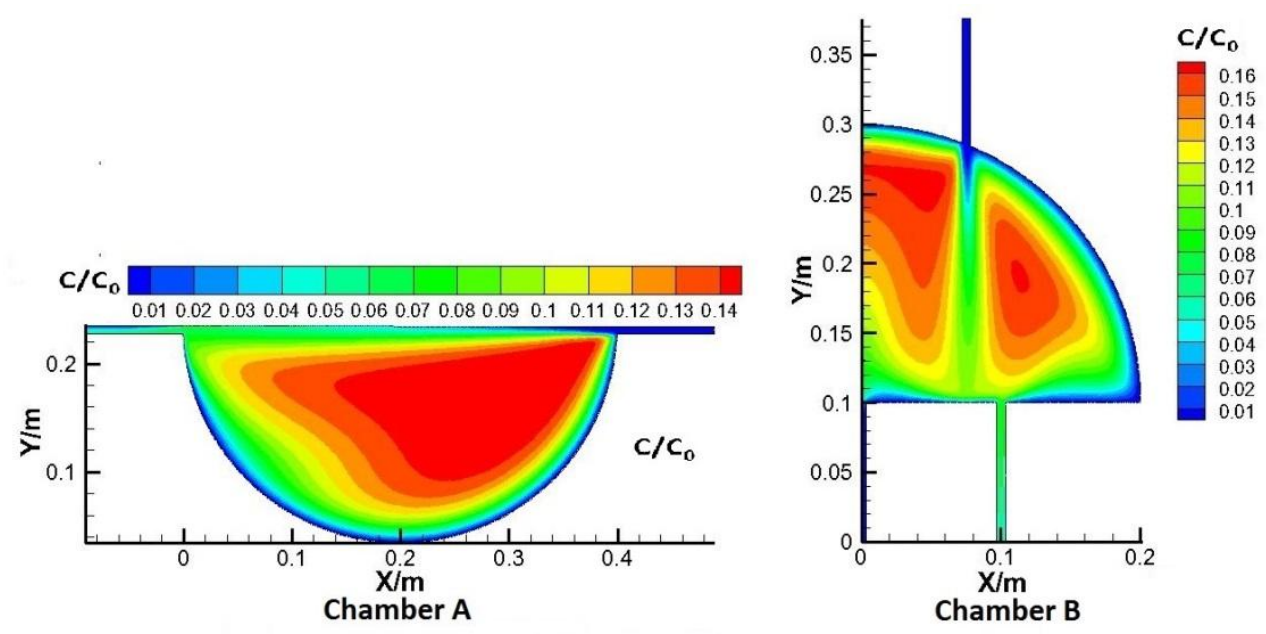

(a) At one minute
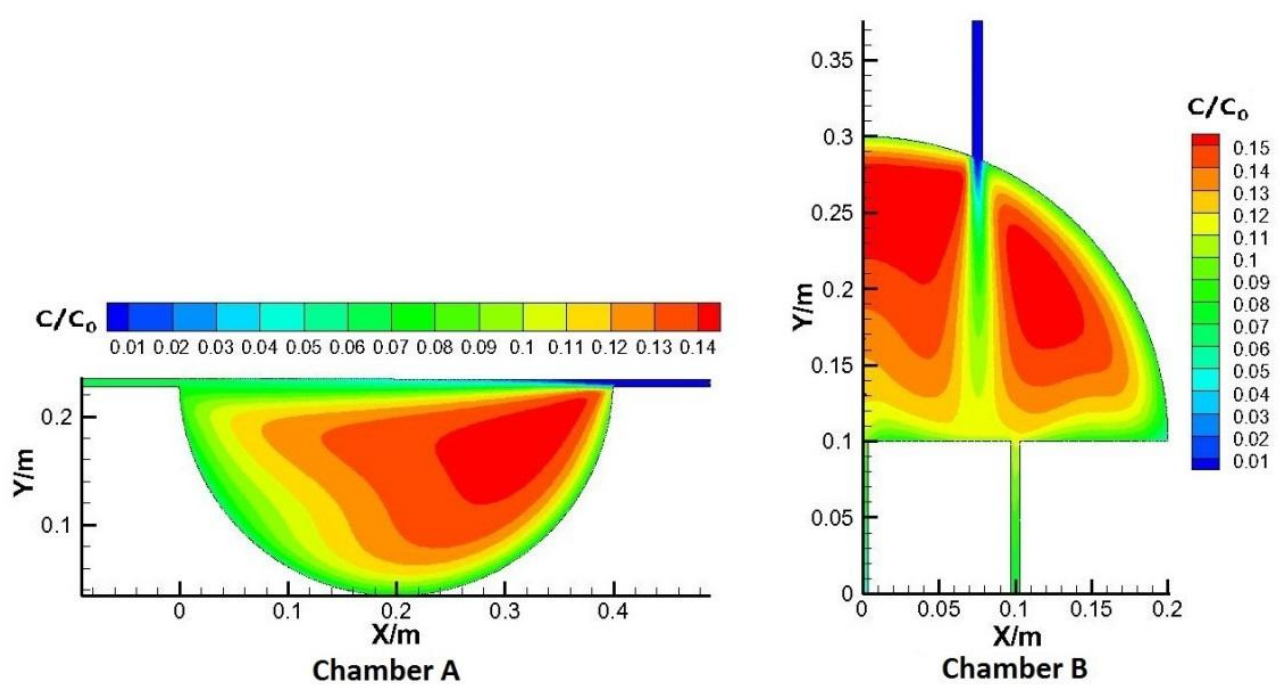

(b) At one hour
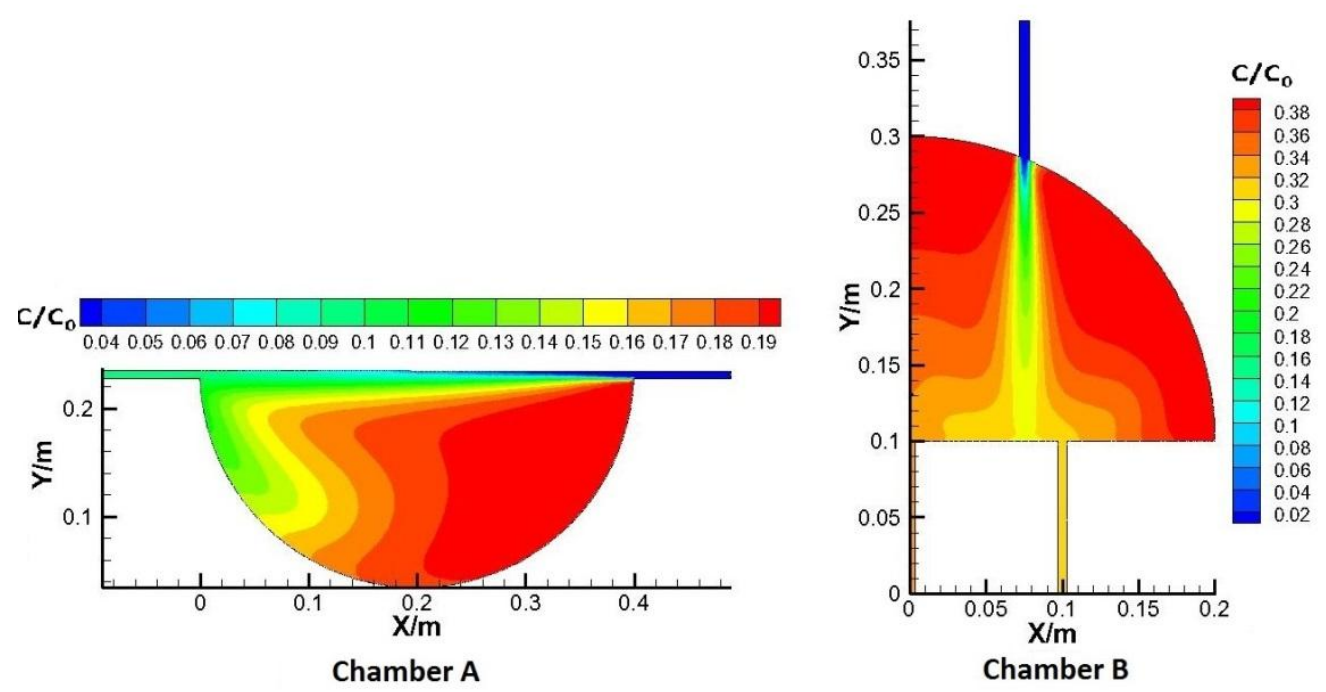

(c) At one day

Figure 6. Non-dimensional concentration $\left(\mathrm{C} \mathrm{C}_{0}\right)$ contour varying against time 


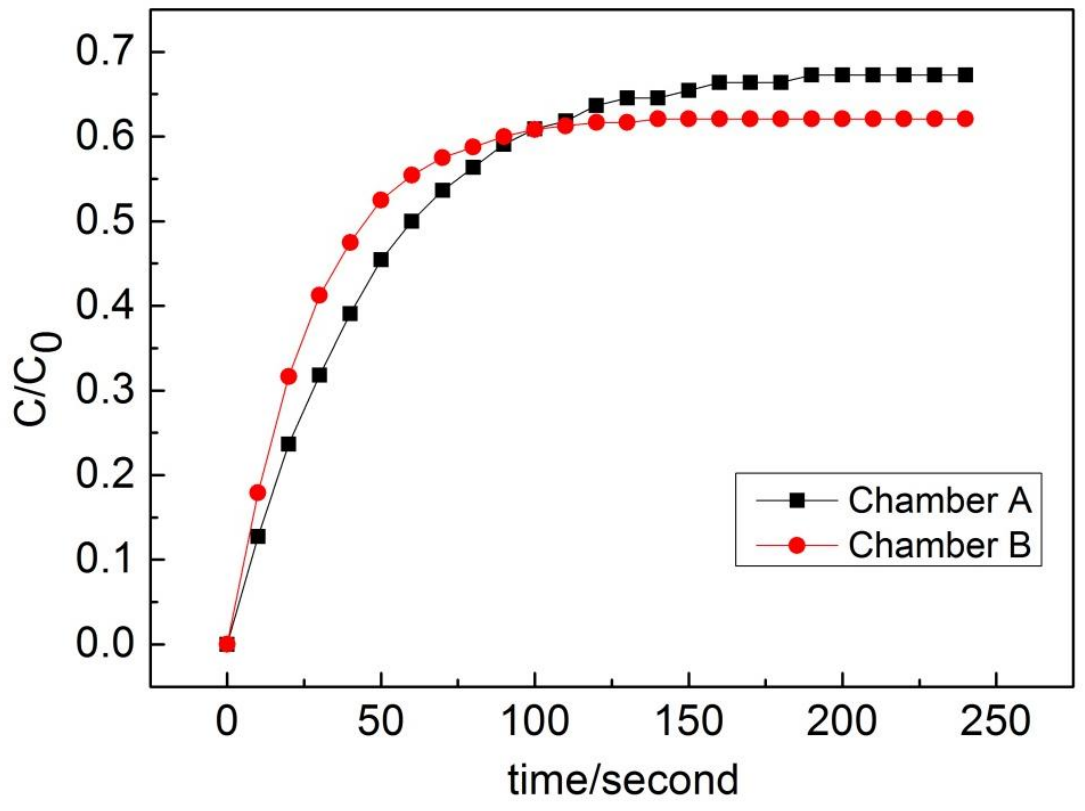

Figure 7. SVOC concentration without sorption varying against time 


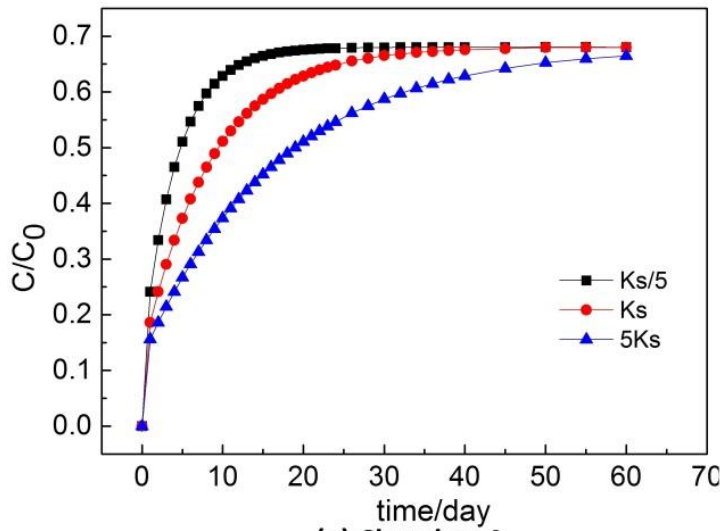

(a) Chamber A

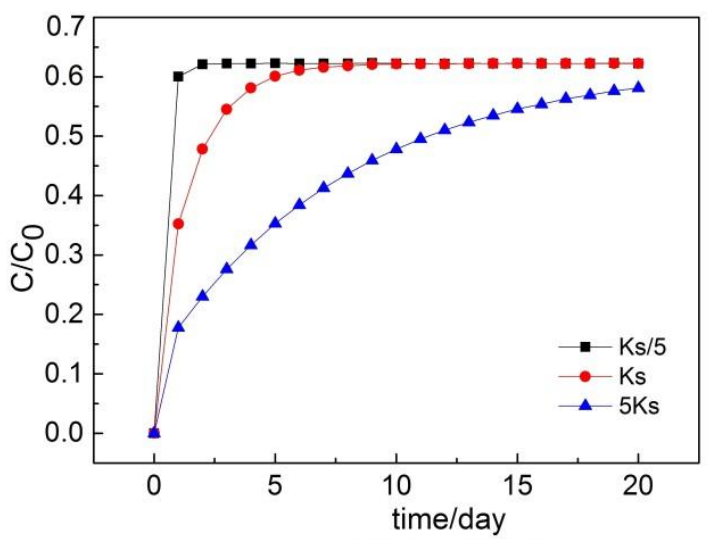

(b) Chamber B

Figure 8. The effect of $K s$ on non-dimensional concentration $\left(C / C_{0}\right)$ 


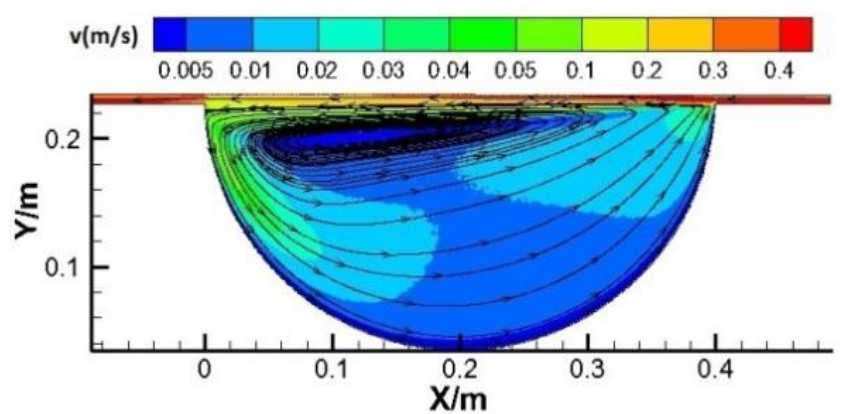

(a) Chamber $\mathrm{A}$

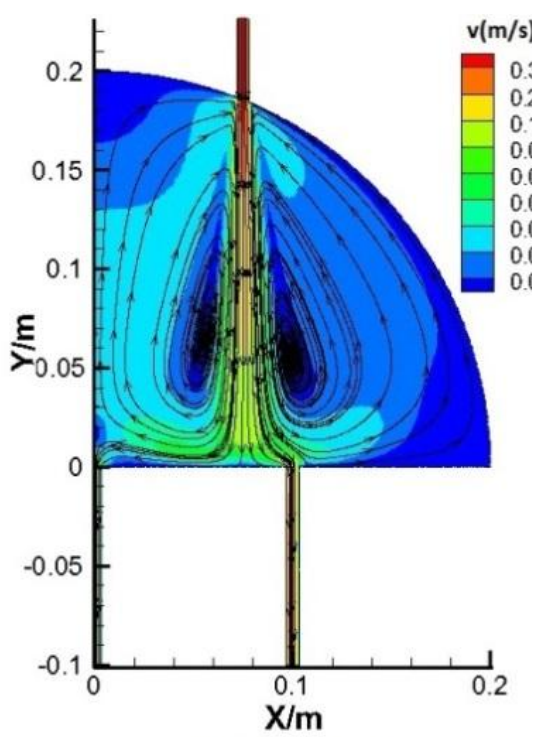

(b) Chamber B

Figure 9. Velocity field in SVOC chambers A and B, respectively 


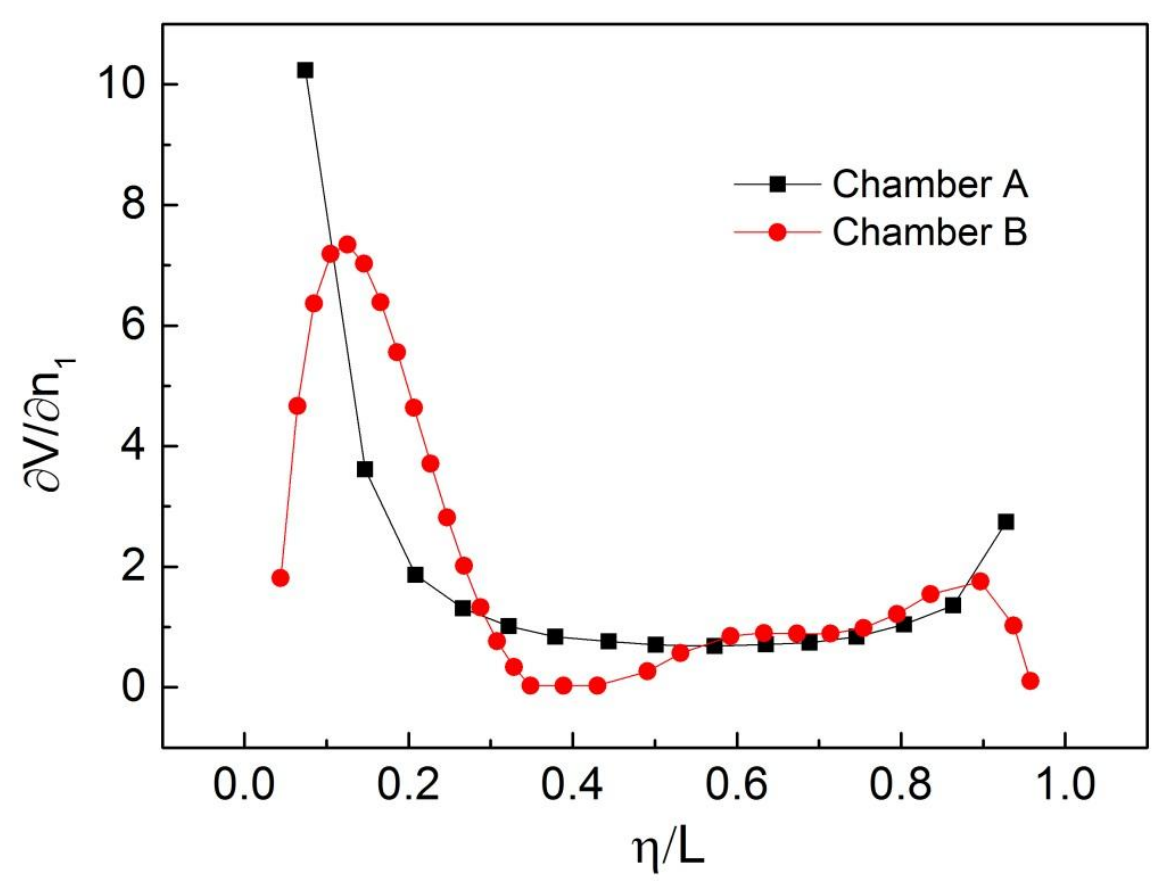

Figure 10. Velocity gradient on the sorption surface 


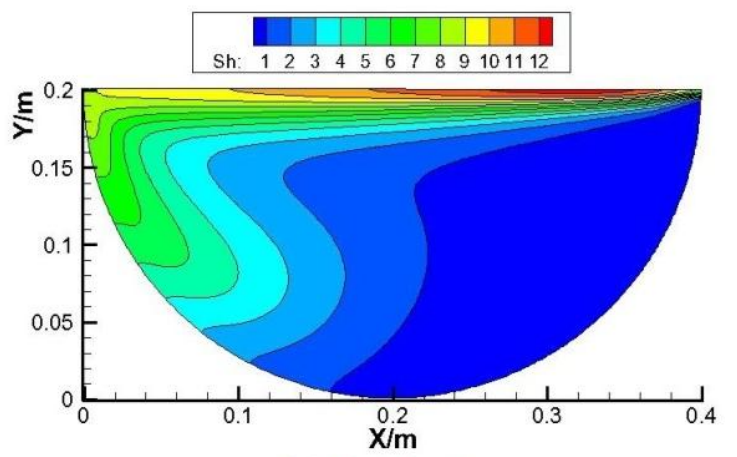

(a) Chamber A

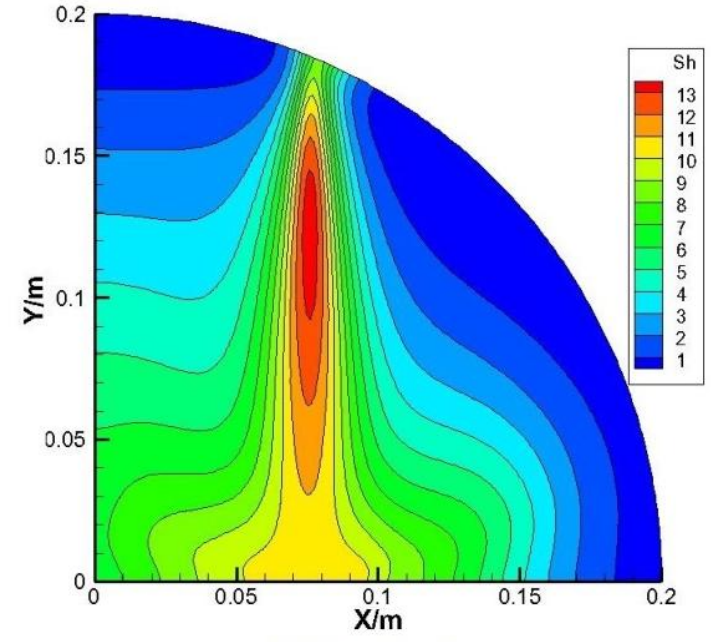

(b) Chamber B

Figure 11. Local Sherwood number on the source surface 


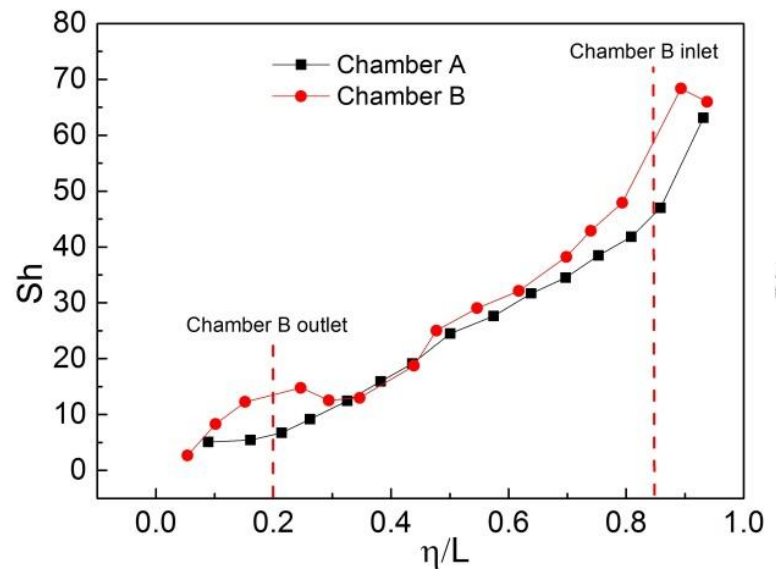

(a) Sh at 1 minute

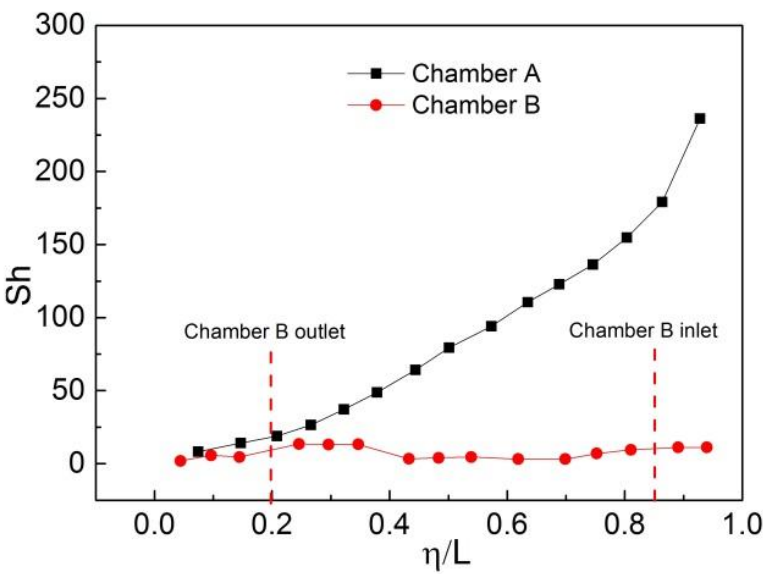

(b) Sh at 1 hour

Figure 12. Local Sherwood number on the sorption surface at (a) 1 minute and (b) 1 hour 